\title{
ATLAS Upgrade for the Super LHC Meeting the challenges of a ten-fold increase in collisions
}

\author{
Kunihiro Nagano,a, on behalf of the ATLAS Collaboration \\ ${ }^{a}$ KEK, 1-1 Oho, Tsukuba, Ibaraki 305-0801 Japan
}

\begin{abstract}
With the Large Hadron Collider (LHC) about to start collisions, plans are already advancing for an upgrade. In this super-LHC (sLHC) project, about ten times the LHC luminosity in some 8 years from now is aimed. Coping with the high instantaneous and integrated luminosity will require many changes to the ATLAS detector. The designs are developing rapidly for an all-new inner-tracker, big changes in the calorimeter and muon systems, as well as improved triggers. This manuscript summarises the environment expected at the sLHC and the status of the improvements to the ATLAS detector.
\end{abstract}

Key words:

ATLAS upgrade, LHC upgrade, sLHC

\section{Introduction}

The Large Hadron Collider (LHC) yields proton-proton collisions and is just starting up with first physics runs planned with $\sqrt{s}=10 \mathrm{TeV}$ in year 2009. The designed maximum instantaneous luminosity, $\mathcal{L}_{\max }$, is $10^{34} \mathrm{~cm}^{-2} \mathrm{~s}^{-1}$. The performance of the LHC is planned to evolve by improving collimators, reaching to the design $\mathcal{L}_{\text {max }}$ around year 2013. The ATLAS detector [1] is designed to be general-purpose covering a wide variety of physics at LHC. It can be expected that the Higgs and/or some other new particles will be discovered (if they exist) with order of $10 \mathrm{fb}^{-1}$ of data luminosity, which can be delivered in a few years of LHC running.

To establish TeV scale physics firmly after discovery, o it becomes important to make precision measurements of ¿ the discovered new phenomena, and also to extend and త continue searches for new phenomena that have low rate or higher mass scale [2]. Thus, it is planned to upgrade the LHC so as to bring a significant increase in luminosity, aiming for an ultimate goal of $\mathcal{L}_{\max }=10^{35} \mathrm{~cm}^{-2} \mathrm{~s}^{-1}$, i.e. ten-times higher than the original LHC design (called super-LHC, sLHC). This project is planned in two phases. In the first phase ("Phase- 1 ") $\mathcal{L}_{\max }=3 \cdot 10^{34} \mathrm{~cm}^{-2} \mathrm{~s}^{-1}$ is a goal. During a shutdown in winter of year 2013-2014, it is foreseen to upgrade the Linac2 injector to Linac4 and also to upgrade the interaction region (IR) by installing new larger-aperture focusing-quadrupoles which will allow the beam size $\beta^{*}$ to reduce from 55 to $25 \mathrm{~cm}$. About few hundreds $\mathrm{fb}^{-1}$ of luminosity will be delivered with about 8 years of operation by the end of the Phase- 1 . Then, the

\footnotetext{
*Corresponding author. Tel.: +81-29-879-6078.

Email address: knagano@post.kek.jp (Kunihiro Nagano)
}

LHC upgrade will enter in the second phase ("Phase-2"). A long shutdown of about 16 months will be devoted to this upgrade. Installation of new injectors and upgrades of IR are anticipated. The goal of the ATLAS experiment in the Phase- 2 is to collect data of about few thousands $\mathrm{fb}^{-1}$ of luminosity.

The ten-fold increase in collisions will however give many challenges to the experiments. The number of minimum bias events per bunch-crossing will reach about 400 events overlaid on each other. The high occupancy in the tracking detectors may degrade the performance. And the detectors and front-end (FE) electronics will sustain a significantly increased radiation dose. Also, low-energy neutrons and photons can cause significant backgrounds in the muon chambers. The trigger and DAQ systems should handle much higher event rates. The ATLAS collaboration is therefore planning modifications to cope with severe conditions at sLHC. Most of the detector components will cope well, for example, both the toroidal and solenoidal magnet systems will remain unchanged. Many research and development $(\mathrm{R} \& \mathrm{D})$ work are rapidly progressing towards defining a letter of intent (LoI). Below, options and ideas for the ATLAS upgrade and relevant preliminary R\&D works are described.

\section{Inner Detector upgrades}

The ATLAS Inner Detector (ID) utilises silicon technology of pixels (Pixel) and strips (SCT) and transition radiation technology (TRT). It covers $\eta<2.5$ and is located in a $2 \mathrm{~T}$ solenoidal field. The most significant upgrade for ATLAS will be the full replacement of the whole ID, foreseen at the Phase-2. The Pixel and SCT will exceed the 
design radiation-hardness of $730 \mathrm{fb}^{-1}$ even if the Phase2 upgrade does not occur and thus requires replacement anyway $^{1}$. The TRT will become difficult to operate due to large dead time caused by expected high occupancy.

An upgrade option being considered is an all-silicon inner tracker. In order to maintain good pattern recognition performance, the granularity is being optimised to keep the hit occupancy less than 1\%. At the same time, efforts are underway to minimise the amount of materials by investigating technologies for powering, construction techniques etc. Simulation studies are in progress to define the layout. Fig. 1 shows the "strawman" layout which has been set as a starting point for evaluating the performance of different layout options. In the central region it has four pixel layers, three layers with short strips and two layers of long strips.

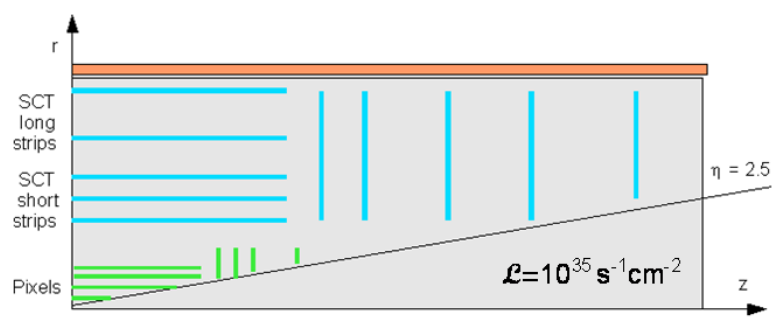

Figure 1: The "strawmann" Inner Detector layout.

\subsection{Pixels}

In the strawman layout, the inner-most layer (called $b$-layer) is at $r=5 \mathrm{~cm}$ and the outer three layers extend to $r=28 \mathrm{~cm}$. The pixel size in the inner two layers is $50 \mu \mathrm{m} \times$ $200 \mu \mathrm{m}$ and twice as long in the outer two.

New technologies are under investigation for the $b$-layer which will be put under the most severe radiation condition. Several R\&D studies are underway: three-dimensional silicon sensors, thin silicon combined with three-dimensional interconnects, gas over slim silicon pixels (GOSSIP), and diamond semiconductors. Prototypes have already been produced and radiation tested for some of these sensor technologies. For example, a full-sized prototype of the diamond detector was successfully operated in a test-beam, resulting in a signal-to-noise ratio of 24 after being irradiated up to an equivalent of a 5-year integrated dose at sLHC [4]. In the three-dimensional sensor technology, the different geometrical arrangement of anodes and cathodes give better charge collection efficiency by reducing electron drift length even after heavy irradiation. An option with $\mathrm{p}-\mathrm{n}$ pitch of $50-100 \mu \mathrm{m}$ is being considered [3].

For the outer layers beyond the $b$-layer, planar pixel sensors are being considered. The read-out architecture and the front-end chips are under development with $130 \mathrm{~nm}$ CMOS technology.

\footnotetext{
${ }^{1}$ Design limits in terms of radiation-hardness discussed in this manuscript are based on current estimates of backgrounds with assuming safety factors of typically $5-10$.
}

\subsection{Strips}

An option being studied is to switch from the current pin-n sensor technology to the n-in-p sensor technology [5]. The n-in-p sensors are expected to be more radiation hard and also do not require full depletion which may not be achieved at high dose.

Also, mechanics and assembly schema are under study. A complete ID system will be constructed to allow a rapid installation, as shown in Fig. 2.

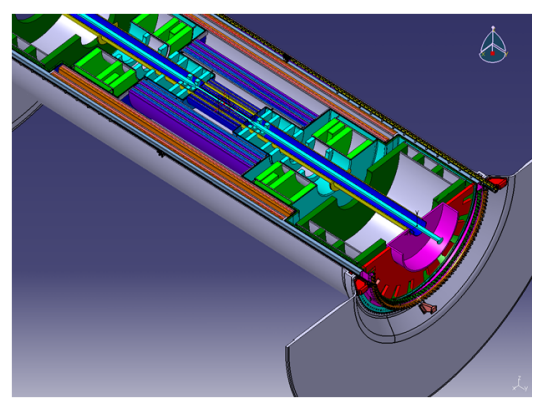

Figure 2: A schematic drawing of the upgraded Inner Detector.

\section{Calorimeter upgrades}

The ATLAS calorimeters use liquid argon (LAr) technology for the electromagnetic calorimetry (Pb-LAr accordion shaped). For hadronic calorimetry, tiles of iron and scintillator are used for the barrel region and $\mathrm{Cu} / \mathrm{W}$ LAr is used for the forward region. While it is expected that the scintillating tiles will sustain the anticipated radiation, one possible limiting factor for LAr technology is the buildup of ions in the drift gap, which may modify the signal amplitude. Preliminary results from a comprehensive test-beam at Protvino show that signal degradation due to space charge effects could only occur at the forward calorimeter (FCAL) located at $3.1<\eta<5$. Also, an additional problem to FCAL could be beam heating in the LAr, as a simulation study shows that a heat load of about $400 \mathrm{~W} / \mathrm{m}^{2}$ can be possible. Thus, an upgrade option being discussed is either to replace the FCAL by a detector with small gap size or to shield the FCAL by installing a warm mini-calorimeter in front of it. Fig. 3 depicts the warm mini-calorimeter option for the calorimeter upgrade.

Also an issue is the cold electronics of the hadronic endcap calorimeter which may be necessary to be replaced due to radiation. And, the readout of the calorimeter is also planned to be upgraded to avoid using an analogue pipeline on the detector. All the signals will be digitised by a very fast $\mathrm{ADC}$ on the FE-board and be sent off detector via fast optical link.

\section{Muon spectrometer upgrades}

The ATLAS muon spectrometer uses the monitored drift tubes (MDT) and cathode strip chambers (CSC) for 


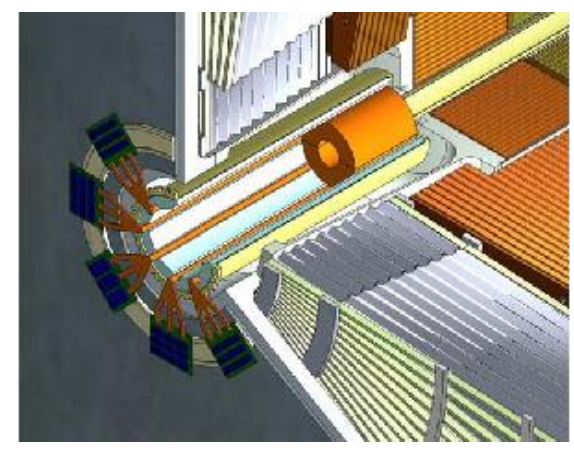

Figure 3: Design of the warm mini-calorimeter in front of the FCAL.

precision tracking, while the resistive plate chamber (RPC) and the thin-gap chambers (TGC) are used primarily for trigger and also supplementary for tracking.

At sLHC, the muon spectrometer is exposed to a very high level of thermal neutron- and photon-backgrounds. Because the amount of such backgrounds is quite uncertain, in a very worst case it might happen that the occupancy exceeds $30 \%$ in most muon chambers at sLHC. It is thus mandatory to understand such backgrounds using the real data of $\mathrm{LHC}$ in order to determine an upgrade scenario.

If the current background estimates are correct only the forward region of the muon system needs to be upgraded. A candidate option is to replace the current MDT and CSC with the MDT with smaller tubes with radius reduced from 30 to $15 \mathrm{~mm}$. About 8 times less space charge is expected owing to smaller cross section of the tube [6]. Alternative options are to do both triggering and precision readout for tracking with a single chamber. Technologies under study are Micromega, GEMs, and TGCs as used in the forward trigger system but optimised for high rate operations.

In order to reduce the amount of background itself, there is also an option under discussion which is to replace the beam-pipe in the region of the end-cap calorimeter and toroid. The current beam-pipe is made of stainless steel which can be activated. By replacing it with beryllium out to $Z=16 \mathrm{~m}$, the backgrounds in the muon chambers will be reduced by typically about $40-60 \%$.

\section{Trigger and DAQ upgrades}

The ATLAS trigger and DAQ (TDAQ) system consists of three levels; the Level-1 is a synchronised hardwarebased trigger, while the higher levels are software based. The Level-2 trigger reduces the fiducial volume necessary to be readout using geometry information at Level-1. And the Event Filter trigger makes use of full detector information with full granularity.

It is anticipated that the TDAQ at SLHC will be challenging as it must cope with the increased event-rates which will evolve rapidly with the increased luminosity as well as with the increased event-sizes which are due the increased number of read-out channels, hit occupancies etc. An example of difficulties in the trigger is muon-trigger rates at Level-1. Fig. 4 shows the inclusive muon trigger rates expected for $\mathcal{L}_{\text {max }}=10^{34} \mathrm{~cm}^{-2} \mathrm{~s}^{-1}$. It is seen that the Level-1 rate is more or less saturated at high $p_{T}$ threshold of $p_{T} \gtrsim 20 \mathrm{GeV}$ due to a limited momentum resolution, and thus raising trigger thresholds on muon $p_{T}$ will not give a good reduction of rates at Level-1.

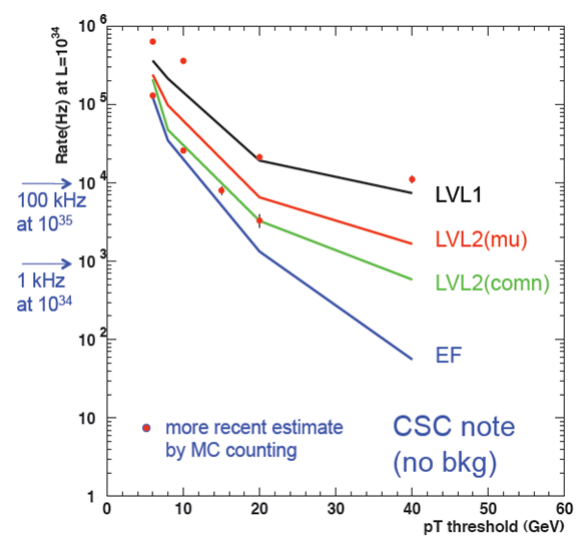

Figure 4: Inclusive muon-trigger rate expected with $\mathcal{L}_{\max }=10^{34}$ $\mathrm{cm}^{-2} \mathrm{~s}^{-1}$.

An extensive discussion towards defining designs of the TDAQ upgrade for sLHC has just begun. Optimisation has to be done with various possible parameters for example the Level-1 trigger latency and data size etc. There are various new trigger schema as well as new architecture designs being considered; for example, track trigger at Level-1, combined objects trigger at Level-1, associative memory track finder, and Level-1.5 trigger scheme to allow quick rejection, etc. A LoI and subsequent R\&D proposals are foreseen around year 2010 .

\section{Summary}

ATLAS detector should keep or improve the performances so as to be sensitive to the rich physics potentials brought by significant increase of luminosity at sLHC. Many detector R\&D works have been actively progressing towards defining a baseline of the upgraded ATLAS detector. A Letter-of-Intent followed by a Technical Proposal, is foreseen in the coming few years.

\section{References}

[1] ATLAS Collaboration, JINST 3 (2008) S08003;

[2] For example, M. Mangano talk presented in sLHC Kickoff meeting, 8-9 April 2008, CERN,

F. Gianotti et al., Eur. Phys. J. C 39 (2005) 293;

[3] E. Tuovinen contribution to these proceedings, U. Parzefall contribution to these proceedings;

[4] M. Cristinziani contribution to these proceedings;

[5] Y. Unno contribution to these proceedings;

[6] A. Engl contribution to these proceedings. 